\title{
Cutting the fat: Artificial muscle oscillators for lighter, cheaper, and slimmer devices
}

\author{
Benjamin M. O’Brien ${ }^{\mathrm{a}, \mathrm{i}}$, Samuel Rosset ${ }^{\mathrm{b}}$, Herbert R. Shea ${ }^{\mathrm{b}}$, Iain A. Anderson ${ }^{\mathrm{a}, \mathrm{c}}$ \\ a'Biomimetics Laboratory, Auckland Bioengineering Institute, The University of Auckland, \\ New Zealand \\ ${ }^{b}$ Microsystems for Space Technologies Laboratory, Ecole Polytechnique Fédérale de Lausanne, \\ Switzerland \\ 'Department of Engineering Science, Faculty of Engineering, The University of Auckland, \\ New Zealand
}

\begin{abstract}
Artificial muscles based on dielectric elastomers show enormous promise for a wide range of applications and are slowly moving from the lab to industry. One problem for industrial uptake is the expensive, rigid, heavy and bulky high voltage driver, sensor and control circuitry that artificial muscle devices currently require.

One recent development, the Dielectric Elastomer Switch(es) (DES), shows promise for substantially reducing auxiliary circuitry and helping to mature the technology. DES are piezoresistive elements that can be used to form logic, driver, and sensor circuitry. One particularly useful feature of DES is their ability to embed oscillatory behaviour directly into an artificial muscle device.

In this paper we will focus on how DES oscillators can break down the barriers to industrial adoption for artificial muscle devices. We have developed an improved artificial muscle ring oscillator and applied it to form a mechanosensitive conveyor. The free running oscillator ran at $4.4 \mathrm{~Hz}$ for 1056 cycles before failing due to electrode degradation. With better materials artificial muscle oscillators could open the door to robots with increased power to weight ratios, simple-to-control peristaltic pumps, and commercially viable artificial muscle motors.
\end{abstract}

Keywords: Artificial Muscle, Dielectric Elastomer, Switch, Actuator, Ring Oscillator, Conveyor

\section{INTRODUCTION}

Mechatronic devices are traditionally made of hard, discrete components. For example, "smart" actuators are defined as actuators coupled to their drive electronics, sensors, control circuits, and gearboxes. However there is a paradigm shift underway towards a new kind of "smart" actuator, one that is characterised by softness and flexibility, distributed intelligence and sensitivity, and multifunctional operation.

Dielectric elastomer artificial muscles are one example of this ${ }^{1}$; they can be distributed into complex networks ${ }^{2,3}$, can self-sense ${ }^{4-7}$, and are multifunctional ${ }^{8,9}$ with $^{\text {actuator }}{ }^{10,11}$, damper ${ }^{12,13}$ and generator modes ${ }^{14-17}$. Because of these advantages they are slowly making the transition from the lab into reality: for example Bayer Artificial Muscle

\footnotetext{
i ben.obrien@auckland.ac.nz
}

Electroactive Polymer Actuators and Devices (EAPAD) 2012, edited by Yoseph Bar-Cohen, Proc. of SPIE Vol. 8340, 834008 - (C) 2012 SPIE · CCC code: 0277-786X/12/\$18 · doi: 10.1117/12.915117 
Incorporated is selling artificial muscles for haptics ${ }^{18}$, Danfoss PolyPower is mass producing artificial muscle material ${ }^{19}$, and Optotune AG is selling artificial muscle laser speckle reducers ${ }^{20}$.

Commercial realities dictate strong focus on narrow niches in the first instance, before easing to allow growth in side markets. It is our view that artificial muscles are being held back from these side markets by the need for discrete and centralized sensor, driver and control circuitry that firstly adds significant expense and secondly goes against the soft smart paradigm shift mentioned earlier.

One way to address this limitation is with Dielectric Elastomer Switch(es) (DES) ${ }^{21-24}$. DES are piezoresistive elements electrodes that change resistance when stretched. As shown in Figure 1 DES can be used to directly charge DEA, and as DEA can deform DES, the two technologies can be used to form arbitrary circuits. DES show promise for alleviating the commercial bottleneck because firstly they can be made out of cheap materials (the same materials used to make DEA electrodes), and secondly they are a form of circuitry that is soft, lightweight, and can be distributed throughout an artificial muscle device.

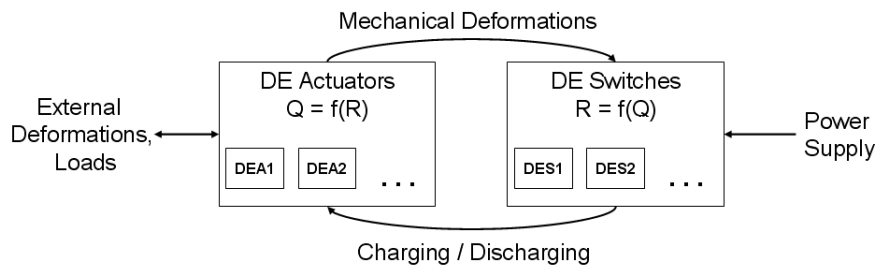

Figure 1: Dielectric elastomer switches and actuators can be combined to form complex circuits.

Many DEA applications only require simple oscillation to work such as rotary motors ${ }^{25,26}$, pumps ${ }^{27}$, and robot locomotion $^{2,28}$. A variety of DES oscillators have been published; we have used a DES oscillator to roll a ball around some rails ${ }^{21}$, developed an artificial muscle ring oscillator $^{23}$, and presented a self-commutating artificial muscle rotary motor $^{24}$. In this paper we show how an artificial muscle ring oscillator can be applied to a mechanosensitive conveyor application. To do this we refine the oscillator design, apply it to the conveyor, characterize it, and discuss its application to industry.

\section{MATERIALS AND METHODS}

As shown in Figure 2 ring oscillators are formed by connecting an odd number of inverters into a ring (see McNeil and Ricketts for a good introduction ${ }^{29}$ ). Ring oscillators have the advantages that they self-start, do not require a mass-spring system or mechanical instability to operate, and when made of artificial muscles they can be directly coupled to applications to confer mechanosensitivity.

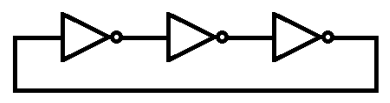

Figure 2: Three stage ring oscillator. Three inverters connected into a ring are unstable and will oscillate as fast as they can.

The oscillator in this paper was formed on a single membrane of 3.5 times equibiaxially pre-stretched VHB 4905 adhered to a $170 \times 170 \mathrm{~mm}$ Perspex frame with three windows cut into it as shown in Figure 3. Within each window sat an artificial muscle inverter (Figure 4) with an actuator and switch on either side of a long and narrow coupling point. 
When the actuator expanded the switch was compressed, and since it was part of a voltage divider circuit (Figure 5) the output of the inverter dropped low. The inverters were made long and narrow to enforce an approximately uniaxial actuation. A $6.5 \times 30 \mathrm{~mm}$ protuberance was used as a strain amplifier for the switch to increase the gain of the inverter by a factor of 2. This gain amplification works because the length of the switch is half that of the actuator, so for a given displacement the switch will see twice the strain as the actuator. It is important that there is full width membrane to either side of the switch to act as an antagonist for the DEA.

Switches were formed out 5:1 Cabot Vulcan XC72 carbon black to Molykote 44 Medium grease and applied during stretching of the membrane so that they were 1.35 times equibixially pre-stretched at rest. Actuators were formed out of Nyogel $756 \mathrm{G}$ carbon grease. Figure 6 shows the complete equivalent circuit of the oscillator which highlights that another way to think about the design is that DES act as discharge resistances for the next stage ie: switches are connected directly across the next actuator, and when one DEA is activated the next is deactivated.

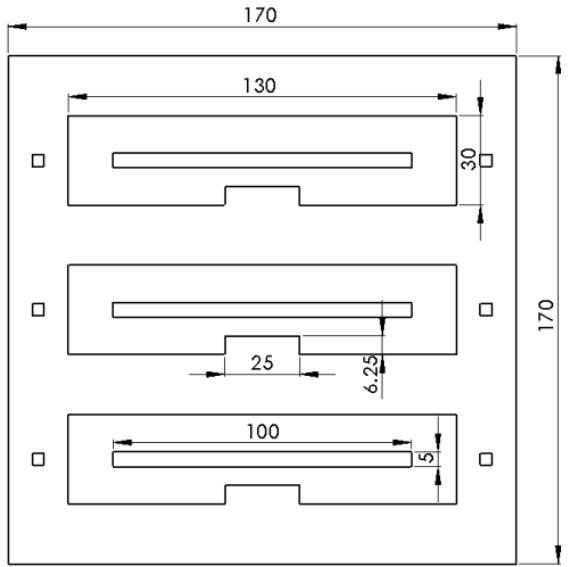

Figure 3: Perspex support frame with three windows cut into it.

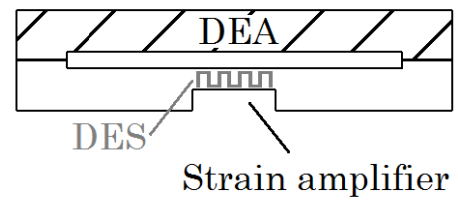

Figure 4: Single inverter unit set into one of the windows of the support frame.

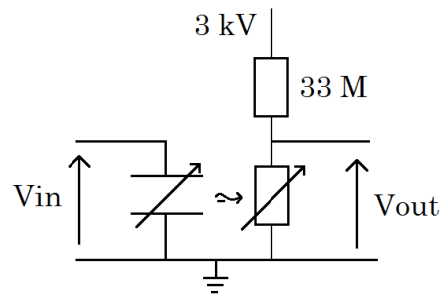

Figure 5: Equivalent circuit of the single inverter.

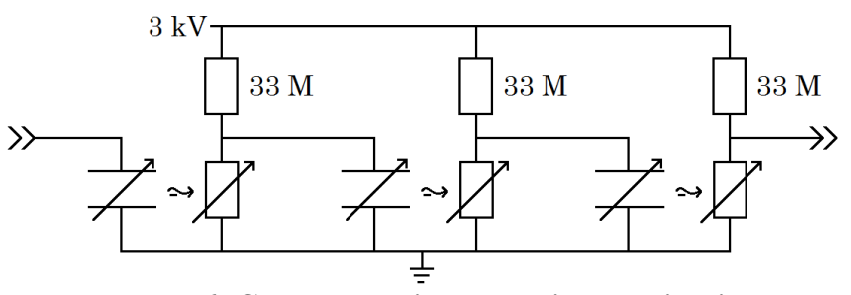

Figure 6: Complete oscillator equivalent circuit

The oscillator was formed into a mechanosensitive conveyor by attaching paper fingers to each coupling point as shown in Figure 7. The paper fingers were bent to point in one direction and this had a ratcheting effect on an emery board (nail file) placed on top of them. This was a mechanosenstive configuration because the load could directly block the passage of mechanical information within the inverters i.e. if a coupling point was blocked from moving then the ring oscillator would stop in a state where the blocked actuator was maximally on, and would start again when the load started to move. It was weakly mechanosensitve however as the paper fingers were compliant and could bend. A more mechanosensitive design would employ stiffer fingers. Figure 8 shows the fabricated conveyor with emery board on top of the fingers. 


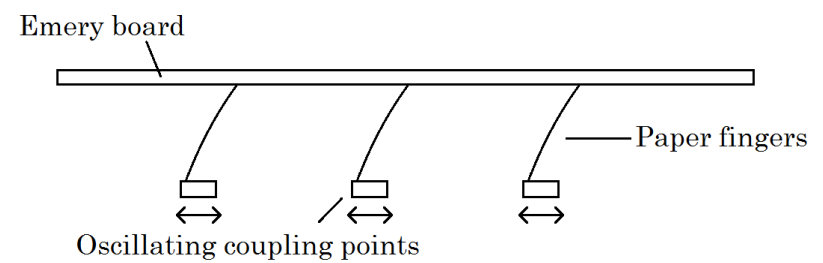

Figure 7: A mechanosensitvie conveyor was made by connecting paper fingers to the inverter coupling points.

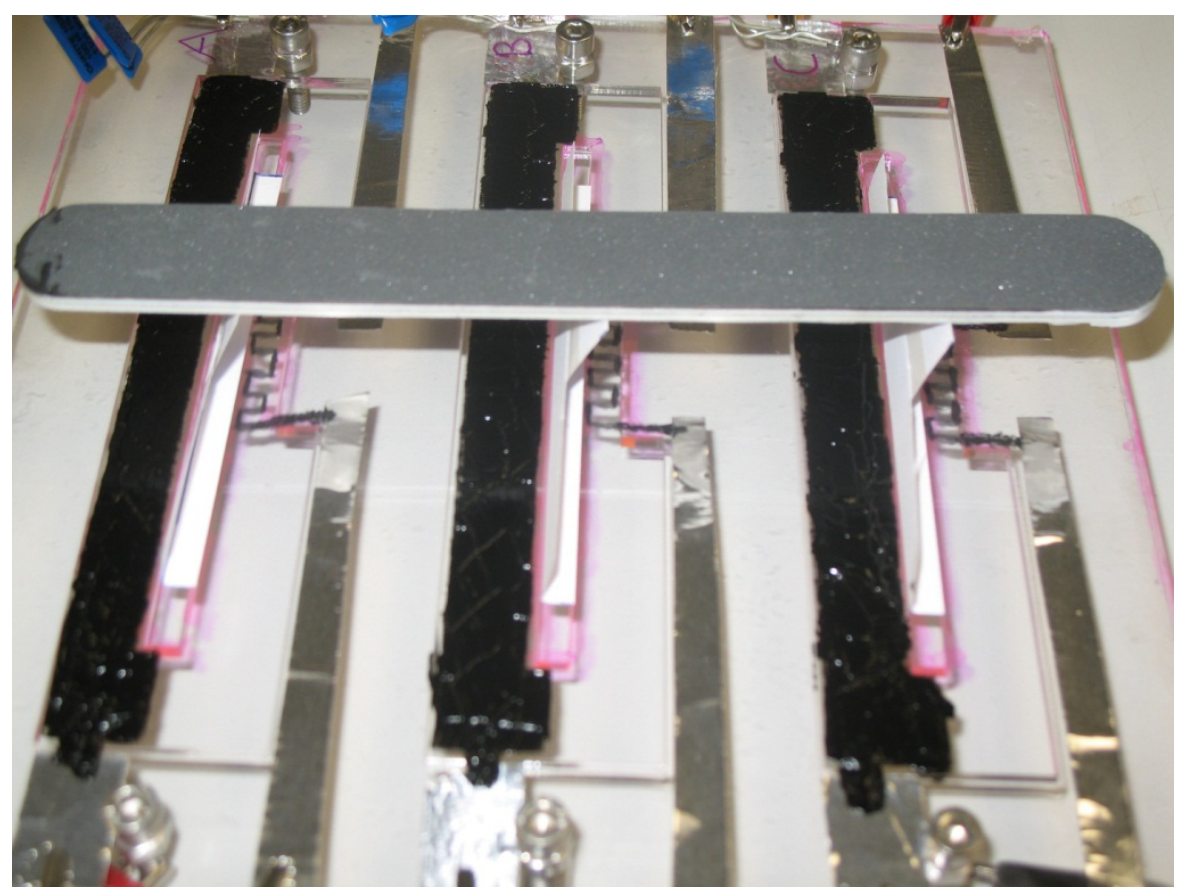

Figure 8: Photo of the conveyor supporting the emery board

\section{RESULTS:}

Figure 9 shows the conveyor carrying the emery board a short distance. To get benchmarking information the emery board was removed and the oscillator was run to failure with its output connected to a high input impedance probe. Start up, stable operation, failure, and period per cycle are shown in Figure 10 to Figure 13. Table 1 shows the consolidated performance data of the oscillator which can be compared to the same performance data for our first ring oscillator ${ }^{23}$. 

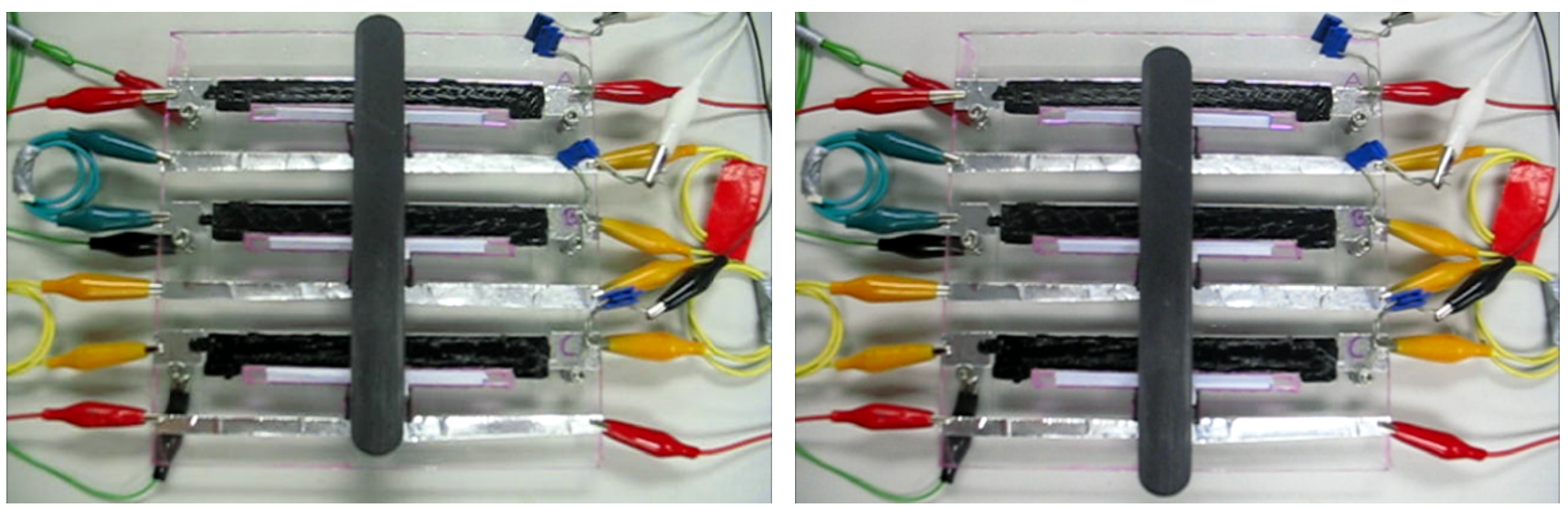

Figure 9: The ring oscillator conveyed the emery board a short distance. Left: Start, Right: Finish.
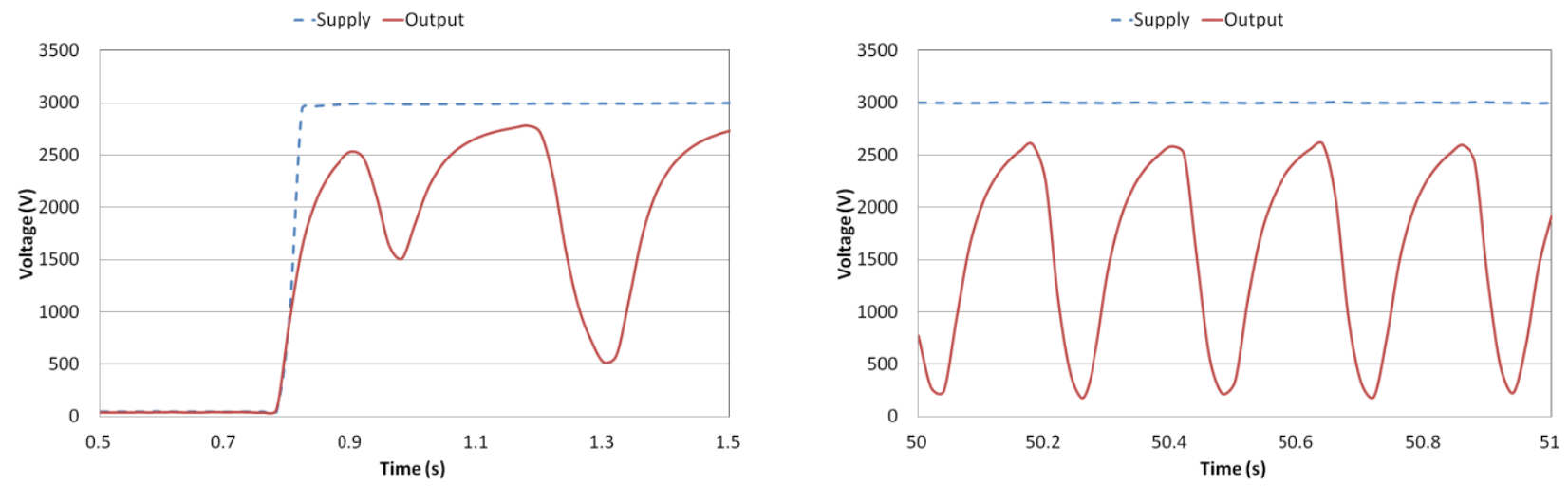

Figure 10: Oscillator startup

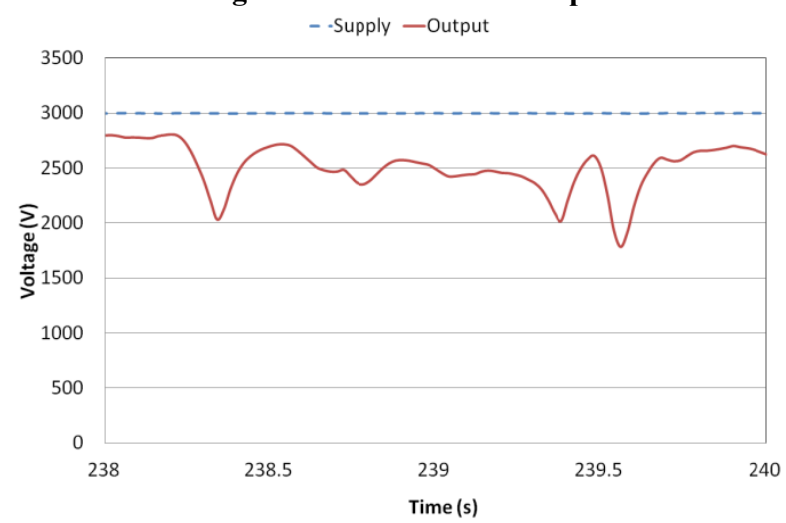

Figure 12: Oscillator decay

Table 1: Oscillator benchmark data

Figure 11: Stable operation at $4.4 \mathrm{~Hz}$

- Period of oscillation

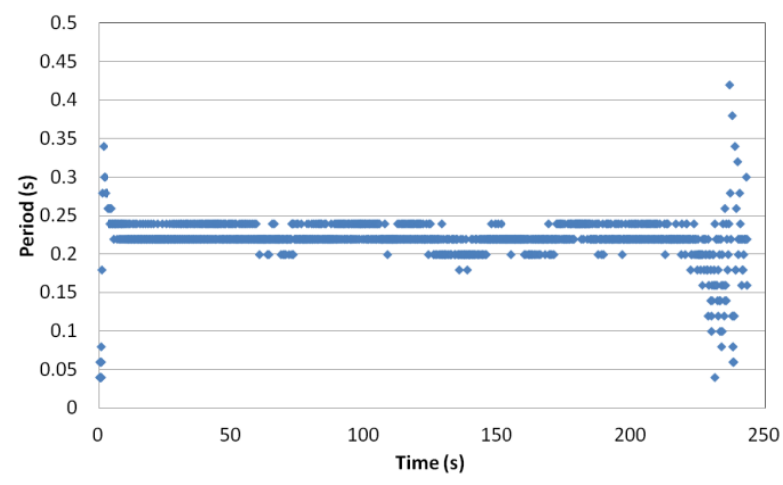

Figure 13: Oscillator period over its lifetime

\begin{tabular}{|r|l|}
\hline Cycles to failure & $\sim 1056$ \\
\hline Frequency of operation & $\sim 4.4 \mathrm{~Hz}$ \\
\hline Average delay per inverter & $\sim 38 \mathrm{~ms}$ \\
\hline Amplitude & $2390 \mathrm{~V}$ (pk - pk), 80\% of supply \\
\hline Waveform & RC sawtooth \\
\hline
\end{tabular}




\section{DISCUSSION}

The conveyor conveyed the emery board a short distance before it stuck with the load simply oscillating back and forth. The reason for this is that the center of gravity of the board moved away from the conveyor causing the board to tip and one finger to stick too well and the other side to not touch at all. Future work includes increasing the number of fingers so that the conveyor can carry things for a longer distance. A mix of smart (actuators coupled to switches) and dumb (no switches) actuator units could also be used to make a longer conveyor, with a few oscillator units acting as pacemakers for the rest. The pacemakers could be clumped together or distributed throughout the array.

During benchmarking the ring oscillator started very quickly and settling into a stable oscillation of $4.4 \mathrm{~Hz}$ within a few cycles. The waveform was a RC sawtooth with the rising side limited by the $33 \mathrm{M} \Omega$ charge resistance, and the falling side running a bit faster due to the on-state resistance of the DES switches. The data presented here was gathered after the ring oscillator had been run for many cycles during the conveyor experiments, thus the lifetime estimate is conservative and the true value may be as much as double the 1056 cycles reported in Table 1 . The oscillator failed due to spark erosion in the switching material, but interestingly there was spark erosion in the interconnects first, which then migrated to the switch. The effects of degradation can been seen clearly in Figure 13 where the oscillator period settles quickly into a stable range which lasts until material ablation becomes too pronounced and the oscillator becomes erratic at the end of its life.

The ring oscillator design presented here is an improvement over our previous design ${ }^{23}$ as it lasted for many more cycles, likely due to the switch strain amplification which allowed the switches to keep working in the presence of greater degradation. It is interesting to note that large improvements in performance can be achieved by simple geometric changes without needing to develop better materials and fabrication processes, although better materials and fabrication processes such as gold ion implantation ${ }^{30-32}$ or other compliant electrodes ${ }^{33,} 34$ are essential for the future of the technology.

Ring oscillators can accelerate the industrial uptake of artificial muscles however they need better materials and fabrication processes to do so. Reliability is especially important, but also sensitivity as commercially available actuator materials can achieve only about $10 \%$ strain. We would like to make a call to the materials science and development community to help us develop better switching materials. As until this happens, dielectric elastomer switches will also remain stuck in the lab. But once it does, artificial muscle oscillators will begin to permeate every aspect of our lives as they eliminate heavy, bulky, and expensive external circuitry, or in other words; as they cut the fat.

\section{ACKNOWLEDGEMENTS}

This work was partially funded by the Royal Society of New Zealand via BM O'Brien's Rutherford Foundation Postdoctoral Fellowship, the Auckland Bioengineering Institute, and from the Swiss National Science Foundation, grant \# 200020-130453. The authors would like to thank S. Akbari, L. Maffli, T. Gisby, T. McKay, S. Schlatter, V. Perret, P. Rosset, S. Pilkington, and M. O'Brien. The authors would especially like to thank S. Astrop for the permanent loan of her emery board.

\section{REFERENCES}

[1] Pelrine, R.E., Kornbluh, R.D., Pei, Q., and Joseph, J.P., "High-Speed Electrically Actuated Elastomers with Strain Greater Than 100\%," Science 287, 836-839 (2000). 
[2] Jung, K., Koo, J.C., Nam, J.-d., Lee, Y.K., and Choi, H.R., "Artificial annelid robot driven by soft actuators," Bioinspiration and Biomimetics 2, S42-S49 (2007).

[3] O'Brien, B.M., Gisby, T.A., Xie, S.Q., Calius, E.P., and Anderson, I.A. "Biomimetic control for DEA arrays," Proc. SPIE 7642 (2010).

[4] Gisby, T.A., Calius, E.P., Xie, S., and Anderson, I.A. "An adaptive control method for dielectric elastomer devices " Proc. SPIE 6927 (2008).

[5] O'Brien, B., Thode, J., Anderson, I., Calius, E., Haemmerle, E., and Xie, S. "Integrated extension sensor based on resistance and voltage measurement for a dielectric elastomer," Proc. SPIE 6524 (2007).

[6] Toth, L.A. and Goldenberg, A.A. "Control system design for a dielectric elastomer actuator: the sensory subsystem," Proc. SPIE 4695 (2002).

[7] Keplinger, C., Kaltenbrunner, M., Arnold, N., and Bauer, S., "Capacitive extensometry for transient strain analysis of dielectric elastomer actuators," Applied Physics Letters 92(192903) (2008).

[8] Pei, Q., Rosenthal, M.A., Pelrine, R., Stanford, S., and Kornbluh, R.D. "Multifunctional electroelastomer roll actuators and their application for biomimetic walking robots," Proc. SPIE 5051 (2003).

[9] Pelrine, R., Kornbluh, R., Carpi, F., De Rossi, D., Kornbluh, R., Pelrine, R., and Sommer-Larsen, P., [Electromechanical transduction effects in dielectric elastomers: actuation, sensing, stiffness modulation and electric energy generation], in Dielectric Elastomers as Electromechanical Transducers. Elsevier, Amsterdam, 1-12 (2008).

[10] Pelrine, R., Kornbluh, R., and Kofod, G., "High-strain actuator materials based on dielectric elastomers," Advanced Materials 12(16), 1223-1225 (2000).

[11] Pelrine, R.E., Kornbluh, R.D., Pei, Q., Stanford, S., Oh, S., Eckerle, J., Full, R., Rosenthal, M., and Meijer, K. "Dielectric Elastomer Artificial Muscle Actuators: Toward Biomimetic Motion," Proc. SPIE 4695 (2002).

[12] Graf, C. and Maas, J. "Electroactive polymer devices for active vibration damping," Proc. SPIE 7976 (2011).

[13] Pelrine, R., Carpi, F., De Rossi, D., Kornbluh, R., Pelrine, R., and Sommer-Larsen, P., [Variable stiffness mode: devices and applications], in Dielectric Elastomers as Electromechanical Transducers. Elsevier, Amsterdam, 141-145 (2008).

[14] McKay, T., O'Brien, B., Calius, E., and Anderson, I., "An integrated, self-priming dielectric elastomer generator," Applied Physics Letters 97(062911) (2010).

[15] McKay, T.G., O'Brien, B.M., Calius, E.P., and Anderson, I.A., "Self-priming dielectric elastomer generators," Smart Materials and Structures 19(5) (2010).

[16] Pelrine, R. and Prahlad, H., [Generator mode: devices and applications], in Dielectric Elastomers as Electromechanical Transducers, F. Carpi, et al., Editors., Elsevier, Amsterdam, 146-155 (2008).

[17] Pelrine, R.E., Kornbluh, R.D., Eckerle, J., Jeuck, P., Oh, S., Pei, Q., and Stanford, S. "Dielectric elastomers: generator mode fundamentals and applications," Proc. SPIE 4329 (2001).

[18] Biggs, S.J. and Hitchcock, R.N. "Artificial muscle actuators for haptic displays: system design to match the dynamics and tactile sensitivity of the human fingerpad," Proc. SPIE 7642 (2010).

[19] Kiil, H.E. and Benslimane, M. "Scalable industrial manufacturing of DEAP," Proc. SPIE 7287 (2009). 
[20] "Application Note: Transmissive Laser Speckle Reducer LSR-3000 \& LSR-OEM Series," Optotune_AG, Dietikon (2011).

[21] O'Brien, B.M., Calius, E.P., Inamura, T., Xie, S.Q., and Anderson, I.A., "Dielectric elastomer switches for smart artificial muscles," Applied Physics A: Materials Science and Processing 100(2), 385-389 (2010).

[22] O'Brien, B.M., McKay, T.G., Xie, S.Q., Calius, E.P., and Anderson, I.A. "Dielectric Elastomer memory," Proc. SPIE 7976 (2011).

[23] O'Brien, B.M. and Anderson, I.A., "An Artificial Muscle Ring Oscillator," Mechatronics, IEEE/ASME Transactions on 17(1), 197-200 (2012).

[24] O'Brien, B.M., McKay, T.G., Gisby, T.A., and Anderson, I.A., "Rotating turkeys and selfcommutating artificial muscle rotary motors," Applied Physics Letters 100(074108) (2012).

[25] Anderson, I.A., Hale, T., Gisby, T., Inamura, T., Mckay, T., O'Brien, B., Walbran, S., and Calius, E.P., "A thin membrane artificial muscle rotary motor," Applied Physics A: Materials Science \& Processing 98, 75-83 (2010).

[26] Anderson, I.A., Tse, T.C.H., Inamura, T., O'Brien, B.M., McKay, T., and Gisby, T., "A soft and dexterous motor," Applied Physics Letters 98(123704) (2011).

[27] Carpi, F., Menon, C., and De Rossi, D., "Electroactive elastomeric actuator for all-polymer linear peristaltic pumps," IEEE/ASME Transactions on Mechatronics 15(3), 460-470 (2010).

[28] Artusi, M., Potz, M., Aristizabal, J., Menon, C., Cocuzza, S., and Debei, S., "Electroactive elastomeric actuators for the implementation of a deformable spherical rover," IEEE/ASME Transactions on Mechatronics 16(1), 50-57 (2011).

[29] McNeill, J.A. and Ricketts, D.S., [The Designer's Guide to Jitter in Ring Oscillators]. The Designer's Guide Book Series, ed. K. Kundert, Springer Science+Business Media, LLC, New York, (2009).

[30] Akbari, S., Niklaus, M., and Shea, H. "Arrays of EAP micro-actuators for single-cell stretching applications," Proc. SPIE 7642 (2010).

[31] Niklaus, M. and Shea, H.R., "Electrical conductivity and Young's modulus of flexible nanocomposites made by metal-ion implantation of polydimethylsiloxane: The relationship between nanostructure and macroscopic properties," Acta Materialia 59(2), 830-840 (2011).

[32] Rosset, S., Niklaus, M., Dubois, P., and Shea, H.R., "Large-stroke dielectric elastomer actuators with ion-implanted electrodes," Journal of Microelectromechanical Systems 18(6), 1300-1308 (2009).

[33] Khodaparast, P., Ghaffarian, S.R., and Khosroshahi, M.R., "Effect of different electrode materials on the performance of smart composite actuators based on dielectric elastomers," Key Engineering Mateirals 334-335, 985-988 (2007).

[34] Kofod, G., Sommer-Larsen, P., Carpi, F., De Rossi, D., Kornbluh, R., Pelrine, R., and SommerLarsen, P., [Compliant electrodes: solutions, materials and technologies], in Dielectric Elastomers as Electromechanical Transducers. Elsevier, Amsterdam, 69-76 (2008). 